\title{
Improving handover of acute orthopaedic admissions
}

\author{
Paul Karayiannis, Jonathan Warnock
}

Ulster Hospital, Belfast, Northern Ireland.

\begin{abstract}
Handover is a crucial part of patient care and is a well recognized cause of patient harm if not performed well. The introduction of full shift working for doctors has placed even more emphasis on this area of patient care. We identified handover of orthopaedic admissions in our unit was substandard. A prospective audit over a one week period including the weekend was undertaken. The Royal College of Surgeons of England guidance on handovers was used as minimum criteria and we also included criteria essential for handover of orthopaedic patients. This initial audit revealed a poor standard of handover. 21 patients were included and in particular patient location (57\%), responsible consultant (14\%), and pending investigations (29\%) were poorly performed. In addition two patient safety incidents were noted, including one admission that was not handed over. To improve the handover we created a trauma specific handover proforma. We then conducted a reaudit again over a one week period including the weekend with the proforma in use. There was a notable improvement, 17 patients were included and only 3 criteria fell below $80 \%$. We presented our findings at the local audit meeting where the results were discussed with all members of the trauma team. We suggested that an electronic proforma, accessible from multiple computers within the hospital may improve handover further. This was created in conjunction with the IT department. Once again we reaudited handover over a one week period including the weekend with the electronic proforma in use. 23 patients were included and a further improvement was noted; only one criterion fell below $95 \%$. In conclusion handover of acute fracture admissions within the unit has undoubtedly improved. The electronic proforma tool was a simple, cost effective, and accurate method of improving handover.
\end{abstract}

\section{Problem}

Handover is now a crucial part of patient care and is a well recognized cause of patient harm if not performed well [1].

The British Medical Association (BMA), the National Patient Safety Agency (NPSA), and the General Medical Council (GMC) [2] have all recognized and highlighted the essential nature of accurate and effective handover. This has further increased in significance as a result of recent changes within medical practice, in particular the introduction of full shift working. In 2007 The Royal College of Surgeons of England produced guidance on handovers, specifically for surgical teams [3].

\section{Background}

The Ulster Hospital in Dundonald is one of the busiest district general hospitals within Northern Ireland. There is an acute and elective general surgical unit as well as a busy Orthopaedic Trauma unit. Seven general surgical and two trauma Senior House Officers (SHOs, comprising Foundation year 2 doctors and core surgical trainees) combine on the rota to cover both specialties out of hours. This 'cross cover' has led to difficulties for SHOs covering two teams overnight and subsequently handing over care of acute admissions to different teams at a similar time each morning.

There are also two other important handovers during each working day. One between the day shift (8am-5pm) SHO to the long-day $\mathrm{SHO}(5 \mathrm{pm}-9 \mathrm{pm})$ and again to the night shift SHO (9pm-8am).

In addition to this some of SHOs covering Trauma had very limited experience of the specialty as their day to day work was in General Surgery. Therefore determining what information was of clinical importance for efficient handover of Trauma admissions was occasionally problematic for these less experienced trainees.

This combination of circumstances led to an informal handover of orthopaedic admissions, often at a substandard level, and commonly patients were not handed over at all. This was undoubtedly a systemic risk to patients and our junior staff and so we aimed to improve the handover of these admissions by creating a proforma tool. This we hoped would not only improve handover and therefore patient safety but give SHO's a template to work from regarding important elements of the patient history or examination relevant to Trauma. In addition recording the handover would also provide valuable data for further audit [4].

\section{Baseline measurement}

As members of the Trauma team we had concerns about the standard of handover from the out of hours cover and its possible impact on patient care. We undertook a prospective audit over a one week period including handover over the weekend. We used the Royal College of Surgeons of England guidance regarding safe handovers as minimum criteria. We also included criteria which we felt was specific and important for acute fracture admissions. The minimum requirements included were; name, date of birth, patient location, responsible consultant, current diagnosis, results, and pending investigations. Criteria we also included were as follows; patient Mini-Mental State Examination (MMSE) and baseline mobility, urgency and frequency of review, management plan, operational issues (e.g. relevant medications such as warfarin/clopidogrel), and any outstanding tasks (e.g. 
echocardiography/Chest X-ray required).

21 admissions were audited in the first week (20/01/15 $26 / 01 / 2015)$. Overall standard of handover was very poor, in particular patient location (57\%), responsible consultant (14\%), results of significant/pending investigations (29\%), co-morbidities potentially complicating surgery (38\%), and outstanding tasks $(38 \%)$ were all well below the expected standard. In addition two patient safety issues were identified; one patient was not handed over and was located by the team in the morning. The other patient suffered a brachial plexus injury following anterior shoulder dislocation was only handed over as a simple dislocation without communication of the nerve injury. In both these cases there was no handover between the $\mathrm{SHO}$ admitting or accepting the patient and the next $\mathrm{SHO}$ on call.

See supplementary file: ds5841.docx - "Baseline Measurement Results"

\section{Design}

To improve this we created a Trauma specific handover proforma which was printed off by the Trauma team every morning. This proforma was initially filled out by Trauma SHOs during the day who then handed it over to the on call SHO. This ensured that all admissions were officially recorded and encouraged effective communication between day and on call teams. This subsequently meant the SHO who had then been on call overnight had a completed list of admissions to present in the morning.

\section{Strategy}

PDSA Cycle 1: Concerns were raised within the Trauma team about lack of formal handover and concern for patient safety. Initial audit of handover of Trauma admissions was undertaken.

PDSA Cycle 2: Given the patient safety issues identified we introduced the handover proforma immediately. SHOs were made aware by email of the changes and by verbal communication by the Trauma team regarding the proforma and expected standard of handover. Initial feedback from the Trauma team and SHOs was positive.

PDSA Cycle 3: We re-audited the handover of Trauma admissions over a one week period including the weekend with the proforma in use (10/02/15 - 16/02/15). 19 patients were included. A notable improvement in the handover of acute fracture admissions was demonstrated. The only criteria to fall below $80 \%$ were Management plan (76\%), Results of Investigations (47\%), and outstanding tasks $(71 \%)$. All patients were handed over and no patient safety issues were identified. Uptake of the paper proforma was $100 \%$.

PDSA Cycle 4: We presented our findings at the local audit meeting. We discussed as a team including consultants, junior staff, and trauma coordinators about the best way to further improve the handover. An electronic version of the proforma was suggested, accessible from multiple computers in the hospital. This would be created in junction with the IT department. The audit and initial reaudit were also presented in poster format at the local patient safety conference were the project was a prize winner.

PDSA Cycle 5: Introduction of dedicated Trauma specific electronic proforma with access from any computer in the hospital with a generic log in. Reaudit of handover over same time period. A further improvement in handover was again noted with positive feedback from admitting SHOs and orthopaedic team regarding continuity of care and overall handover process.

\section{Results}

Following the introduction of the electronic handover proforma, handover was again audited over a one week period (10/06/2015 16/06/2015). 23 patients were included. The only criterion to fall below $95 \%$ was results of pending investigations (82\%). No patient safety issues were identified and uptake of the electronic proforma $100 \%$. It received positive feedback from the Trauma team for maintaining a high standard of care and the cross-covering SHOs who were able to give a concise handover with clinically relevant information.

See supplementary file: ds6226.docx - "Complete Results Graph (1)"

\section{Lessons and limitations}

Any handover is only as good as the individual clinician reviewing and presenting the patient information. No proforma or online system can ever fully replace good communication between professionals. However we have highlighted how some relatively simple adjuncts electronic or otherwise can significantly improve handover and therefore improve patient care and reduce risk. With the introduction of full shift work, the importance of effective handover has undoubtedly increased. We must therefore ensure that this vital part of providing the best possible care for patients is not overlooked.

Creating the proforma also helped SHOs less familiar with Trauma patients quickly identify important priorities for handover of acute admissions. It also provided an enhanced training opportunity for cross covering SHOs who felt better prepared to present the admissions.

Challenges during the audit were mainly setting up a trust wide online proforma accessible on all computers but once we overcame this we produced an extremely effective electronic tool. This will be enforced during changeover periods by the permanent Trauma Coordinators and staff grades ensuring this remains a developing system within the unit.

\section{Conclusion}

Handover of Trauma admissions within the Ulster hospital has undoubtedly improved and reduced patient risk. The electronic 


\section{BMJ Quality Improvement Reports}

handover tool has proven to be a simple, cost effective, and accurate method of improving communication in a complex out of hours system. It is also a highly reproducible quality improvement project that would be applicable to many teams in many specialties.

\section{References}

1 Acute Care Toolkit 1: Handover. London: Royal College of Physicians. May 2011.

2 British Medical Association: Safe handover: safe patients Guidance on clinical handover for clinicians and managers. Junior Doctors committee. London. 2004.

3 Safe Handover: Guidance from the Working Time Directive working party. The Royal College of Surgeons of England. March 2007.

4 Till A, Sall H, Wilkinson J. Safe Handover: Safe Patients - The Electronic Handover system, BMJ Qual Improv Report 2014;2: doi:10.1136/bmjquality.u202926.w1359

\section{Declaration of interests}

Nothing to declare.

\section{Acknowledgements}

Katharine Dane (Lead Trauma Coordinator) and the Trauma and Orthopaedic Surgery Department Ulster Hospital, Dundonald.

\section{Ethical approval}

Ethical approval was not sought for this project, according to local policy within the South Eastern Trust this work met the criteria for operational improvements exempt from ethics review. This work was primarily intended to improve local care and not provide detailed knowledge in the field of inquiry. This works meets the criterion as handover is a universally accepted part of patient care and we simply evaluated improvements in handover as a result of introduction of paper and electronic proforma. 